\title{
Survival and Failure Outcomes in Primary Thyroid Lymphomas: A Single Centre Experience of Combined Modality Approach
}

\author{
Ritesh Kumar, ${ }^{1}$ Divya Khosla, ${ }^{1}$ Narendra Kumar, ${ }^{1}$ Sushmita Ghoshal, ${ }^{1}$ Anjan Bera, \\ Ashim Das, ${ }^{2}$ and Suresh Chander Sharma ${ }^{1}$ \\ ${ }^{1}$ Department of Radiotherapy and Oncology, Regional Cancer Centre, Postgraduate Institute of Medical Education and Research, \\ Chandigarh 160012, India \\ ${ }^{2}$ Department of Pathology, Postgraduate Institute of Medical Education and Research, Chandigarh 160012, India
}

Correspondence should be addressed to Divya Khosla; dr_divya_khosla@yahoo.com

Received 18 June 2013; Revised 7 August 2013; Accepted 12 August 2013

Academic Editor: B. Stack

Copyright (C) 2013 Ritesh Kumar et al. This is an open access article distributed under the Creative Commons Attribution License, which permits unrestricted use, distribution, and reproduction in any medium, provided the original work is properly cited.

\begin{abstract}
Primary thyroid lymphoma (PTL) is a rare malignancy and represents $2 \%-5 \%$ of all thyroid malignancies and $1 \%-2.5 \%$ of all malignant lymphomas. We present our institute's experience in combined modality management of 16 successive patients of PTL treated from 2005 to 2010. The median age of the patients was 56.0 years. Five patients were males, and 11 patients were females. An enlarging thyroid mass was the most common presenting symptom. 14 patients had diffuse large B-cell lymphoma, and 2 patients had follicular lymphoma. The most common stage of presentation was stage II comprising $6(37.5 \%)$ patients. All patients received CCT, and only 12 patients received involved field RT with a median dose of 36.0 Gy. 10 patients (62.5\%) had CR, and 6 patients (27.5\%) had PR. Eight patients had disease progression in subsequent followup and this included the initial 6 patients with PR. The 5-year DFS was $40.0 \%$, and median DFS was 47 months. The 5 -year OS was $41.0 \%$, and median OS was 51 months. Most common presentation in our series was locally advanced tumors. Most of these patients require combined modality management. Risk-adapted and multimodality approach is the need of the hour to achieve good control rates while minimizing treatment related toxicity.
\end{abstract}

\section{Introduction}

Primary thyroid lymphoma (PTL) is a rare malignancy and represents $2 \%-5 \%$ of all thyroid malignancies $1 \%-2.5 \%$ of all malignant lymphomas $[1,2]$. This rare disease usually affects middle- to older-aged individuals with a female predilection and presents with a rapidly enlarging anterior neck mass with or without cervical lymphadenopathy, often leading to compressive symptoms $[3,4]$. PTL is associated with Hashimoto's thyroiditis, and the relative risk of developing malignant lymphoma of thyroid, is greater in patients with Hashimoto's thyroiditis $[5,6]$. Most thyroid lymphomas are B-cell type non-Hodgkin's lymphomas (NHL), and Hodgkin's and T-cell lymphomas are extremely rare [7-10]. The optimal management strategy for PTL remains somewhat controversial. Because of the rarity of PTL, no randomized control trial has compared the efficacy of multimodality versus single modality treatment. Radiotherapy (RT) is a mainstay of treatment as PTL is highly sensitive to radiation. Systemic chemotherapy (CCT) has diminished the likelihood of local and systemic relapses; combination CCT is generally considered, followed by involved field radiotherapy (IFRT). We herein report our institutional experience of 16 successive patients of PTL being treated from 2005 to 2010.

\section{Material and Methods}

2.1. Patient Population and Initial Evaluation. We retrospectively reviewed the patients of primary thyroid lymphomas (PTL) from January 2005 to December 2010 treated in our institute. Total number of patients was 16 . We reviewed the records of these patients to extract the following information: age, sex, clinical symptoms, histology, radiology (CT/MRI), 
TABLE 1: Patient characteristics.

\begin{tabular}{lc}
\hline Total number of patients & 16 \\
\hline Age (in years) & 56.0 \\
Median & $24-68$ \\
Range & \\
Sex & $5(31.3 \%)$ \\
Male & $11(68.7 \%)$ \\
Female & \\
Stage & $4(25.0 \%)$ \\
I & $6(37.5 \%)$ \\
II & $3(18.75 \%)$ \\
III & $3(18.75 \%)$ \\
IV & \\
B-symptoms & $7(43.75 \%)$ \\
Present & $9(56.25 \%)$ \\
Absent & \\
Neck node & $12(75.0 \%)$ \\
Present & $4(25.0 \%)$ \\
Absent & \\
Thyroiditis & $0(0.0 \%)$ \\
Present & $16(100.0 \%)$ \\
Absent & \\
Histology & $14(87.5 \%)$ \\
DLBCL & $2(12.5 \%)$ \\
FL &
\end{tabular}

tumor extent, chemotherapy regimens and doses, radiation (technique, total dose, dose per fraction, and number of fractions), toxicity, response, recurrence, progression, metastases, and death. Staging investigations included medical history and physical examination, complete hematological profile, blood chemistry, chest radiograph, contrast enhanced computed tomography (CECT) of neck, chest, abdomen, and pelvis, and bone marrow aspiration and biopsy.

2.2. Treatment. Surgery was limited to biopsy for histopathological diagnosis. After initial FNAC diagnosed thyroid lymphoma, all patients underwent core biopsy for exact histopathological characterization and flow cytometric studies. CCT and RT were used in the treatment. CCT was given with either Cyclophosphamide, Doxorubicin, Vincristine, and Prednisolone (CHOP) or (CVP) Cyclophosphamide, Vincristine, and Prednisolone regimen in standard doses at three weekly intervals. RT was delivered in conventional 1.8-2 Gy per fraction. RT planning evolved with time and expertise, and patients were planned with two-dimensional (2D), three-dimensional conformal (3DCRT), and intensity modulated (IMRT) techniques.

2.3. Followup. The period between the first complaint and diagnosis was registered as symptom duration. Survival, recurrence, and progression information were collected through chart review, patient, or relative contact. Response evaluation was noted both clinically and radiologically, and RECIST criteria were applied [11].
TABLE 2: Treatment details.

\begin{tabular}{lc}
\hline Treatment approach & \\
CCT + RT & $14(87.5 \%)$ \\
CCT alone & $2(12.5 \%)$ \\
CCT regimen & \\
CHOP & $15(93.75 \%)$ \\
CVP & $1(6.25 \%)$ \\
RT & \\
Dose (median) & $36.0 \mathrm{~Gy}$ \\
Dose (range) & $30-40 \mathrm{~Gy}$ \\
\hline
\end{tabular}

2.4. Statistical Analysis. SPSS v 15 was used for statistical analysis. The Kaplan-meier survival analysis was done for analyzing disease-free-survival (DFS) and overall survival (OS) [12].

\section{Results}

3.1. Patient Characteristics. Patient characteristics are summarized in Table 1. Between January 2005 and December 2010, 16 patients of PTL were registered in our department. The median age of the patients was 56.0 years and ranges from 24 years to 68 years. five patients $(31.3 \%)$ were males and 11 patients $(68.7 \%)$ were females. The median duration of symptoms was 6 months. An enlarging thyroid mass was the most common presenting symptom manifesting in all of the patients followed by aerodigestive tract symptoms, including dyspnea and dysphagia. B-symptoms were present in 7 (43.75\%) patients and neck lymphadenopathy was present in $12(75.0 \%)$ patients. None of the patients had thyroiditis. On histopathological analysis, 14 patients $(87.5 \%)$ had diffuse large B-cell lymphoma (DLBCL) and 2 patients (12.5\%) had follicular lymphoma (FL). The most common stage of presentation was stage II comprising 6 (37.5\%) patients followed by stages I, III, and IV comprising 4 (25.0\%), 3 (18.75\%), and $3(18.75 \%)$ patients, respectively. Out of the three stage IV patients, two had bone marrow involvement, while one had liver involvement.

3.2. Treatment Details (Table 2). Treatment modalities consisted of CCT and RT. All patients received CCT, 15 (93.75\%) patients with $\mathrm{CHOP}$ regimen and one patient with CVP regimen. Median number of cycles was six. 12 patients received involved field localized $\mathrm{RT}$ with a median dose of 36.0 Gy (range 30-40 Gy) in 1.8-2 Gy per fraction. RT was delivered using 2D RT in 7 patients (43.75\%), and 3DCRT in $5(31.25 \%)$ while 2 patients $(12.5 \%)$ did not receive RT. The 2 patients who did not receive RT were young patients with stage II FL histology.

3.3. Treatment Toxicity and Compliance. All patients completed treatment with no significant toxicity or treatment interruption. RT toxicity occurred in 12 patients $(75.0 \%)$ in the form of grade 1-2 dermatitis and mucositis, and there was no grade 3 or higher toxicity. CCT toxicity was seen in 7 patients in the form of grade 1-2 hematological toxicity. 
TABLE 3: Response evaluation at treatment completion.

\begin{tabular}{lc}
\hline Clinical response & \\
Asymptomatic & $10(62.5 \%)$ \\
Improved & $6(27.5 \%)$ \\
Radiological response & \\
CR & $10(62.5 \%)$ \\
PR & $6(27.5 \%)$ \\
\hline
\end{tabular}

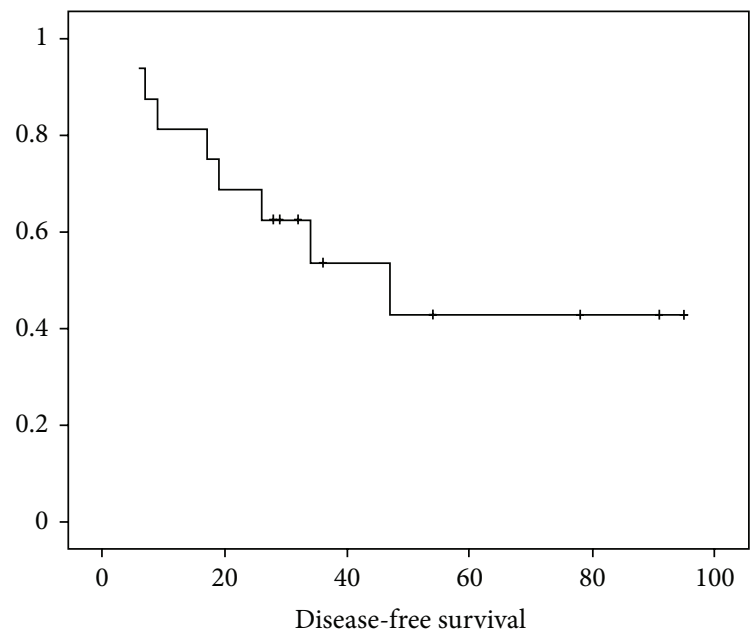

FIgure 1: The Kaplan-Meier curve showing disease free-survival (DFS).

3.4. Clinical Outcomes (Table 3). After treatment completion, patients were assessed for response both clinically and radiologically. 10 patients (62.5\%) were asymptomatic and 6 patients $(27.5 \%)$ had significant improvement in symptoms. As per the RECIST criteria, 10 patients (62.5\%) had CR and 6 patients (27.5\%) had PR. Eight patients had disease progression in subsequent followup, and this included the initial 6 patients with PR. The other 2 patients who initially had CR had recurrence in retroperitoneal lymph node (RPLN) and brain respectively. Out of the eight patients who had disease progression, 5 received second line CCT with MIME regimen comprising mitoxantrone, ifosfamide, and etoposide in standard doses. One patient with recurrence in brain received whole brain $\mathrm{RT}$ in doses of $30 \mathrm{~Gy}$ in 10 fractions. Median duration of followup was 40 months (range 10-95). The 5-year disease free-survival (DFS) of all patients was $40.0 \%$ (Figure 1) and median DFS was 47 months. The 5year overall survival (OS) was $41.0 \%$ and median OS was 51 months (Figure 2). On subgroup analysis, the stagewise median survival was 65 months, 54 months, 29 months, and 8 months for stages I, II, III, and IV, respectively.

\section{Discussion}

Primary thyroid lymphoma (PTL) is a rare malignancy and represents $2 \%-5 \%$ of all thyroid malignancies, $1 \%-2.5 \%$ of all malignant lymphomas, and $2.5 \%-7 \%$ of all extranodal lymphomas $[1,2]$. PTL is commonly seen in middle to older

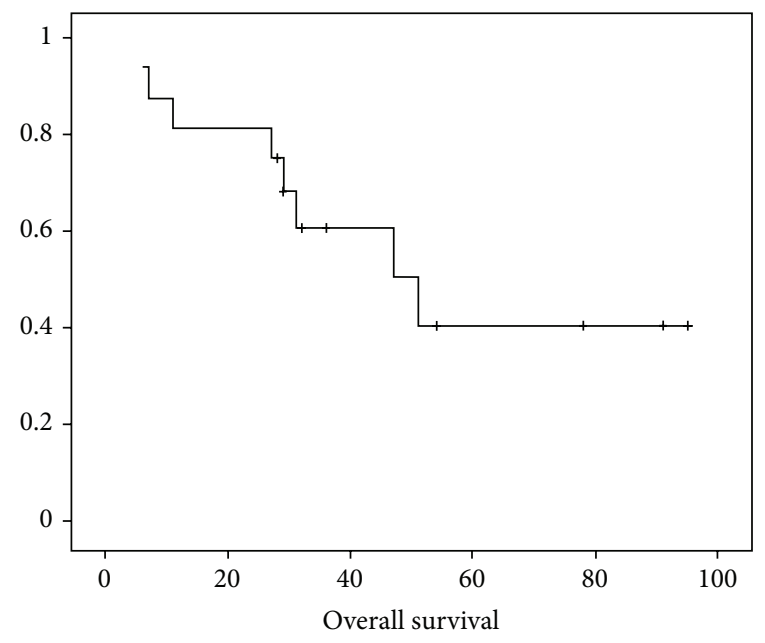

Figure 2: The Kaplan-Meier curve showing overall-survival (OS).

aged women and usually presents as a painless mass in the thyroid gland or diffuse enlargement that causes symptoms related to compression, such as hoarseness, dysphagia, and dyspnea $[3,4]$. In our series, PTL was more common in females (68.7\%), and the most common presentation was diffuse enlargement of thyroid gland followed by compressive symptoms. The presence of B-symptoms is uncommon. PTL is associated with Hashimoto's thyroiditis, and the relative risk of developing malignant lymphoma of thyroid has been estimated to be $40-80$ times greater in patients with Hashimoto's thyroiditis $[5,6]$. However, none of the patients in our series had associated thyroiditis.

Most thyroid lymphomas are B-cell type non-Hodgkin's lymphomas (NHL), and Hodgkin's and T-cell lymphomas are extremely rare [7-10]. The most common B-cell NHL is DLBCL and thyroid DLBCL at a localized stage generally has a good prognosis, but it is heterogenous and sometimes has a poor prognosis [13]. Fourteen patients $(87.5 \%)$ had DLBCL and 2 patients $(12.5 \%)$ had FL in our series.

Ann Arbor staging system is used for staging as in systemic NHL [14]. Most of the patients present with stage I or II disease [15]. In our series, the most common stage of presentation was stage II comprising $6(37.5 \%)$ patients followed by stages I, III, and IV comprising 4 (25.0\%), 3 $(18.75 \%)$ and $3(18.75 \%)$ patients. Out of the three stage IV, patients, respectively, two had bone marrow involvement, while one had liver involvement.

The optimal management strategy for PTL remains somewhat controversial. Because of the rarity of PTL, no randomized control trial has compared the efficacy of multimodality versus single modality treatment. Earlier, local surgery was the primary management strategy because of the difficulty in distinguishing histologically between malignant lymphoma and anaplastic thyroid carcinoma, especially based on preoperative biopsy $[16,17]$. Now, diagnosis can be accomplished using fine needle aspiration biopsy (FNAB) or by core or open biopsy $[18,19]$.

RT is mainstay of treatment as PTL is highly sensitive to radiation. The RT doses delivered varied from 30 to 
50 Gy as reported in different series [20, 21]. To diminish the likelihood of local and systemic relapses, combination chemotherapy is generally considered, followed by involved field radiotherapy (IFRT) [22, 23]. The CCT regimens used commonly are CVP, CHOP, and R-CHOP as in systemic NHL $[24,25]$. In the literature, the 5-year OS rates range from $35 \%$ to $100 \%$. Ha et al. showed 5-year OS and DFS of $64 \%$ and $76 \%$ in a retrospective series and concluded that localized PTL has good prognosis when managed with combined modality treatment [26]. Matsuzuka et al. reported a $100 \%$ OS rate at 8 years for a subset of patients treated with CCT and RT [27]. In a review of 211 patients with PTL, Doria et al. demonstrated that addition of CCT to RT significantly lowered the distant metastases and overall recurrence rates [22]. The 5-year OS in our series was $41 \%$, and these slightly inferior results may be attributed to the 6 patients $(37.5 \%)$ who had stage III, and IV disease

The most important prognostic factors influencing the outcome are age, stage, histopathological subtype, grade, RT, and CCT [28-32]. Local recurrences and distant metastases may develop long after the initial treatment, sometimes after several years, underlining the need for long term followup.

The management of PTL is a paradigm of cooperation between clinicians, surgeons, and pathologists from establishing diagnosis to organizing the therapeutic strategy. With newer targeted chemotherapeutic agents, radiation techniques, diagnostic and imaging facilities, there is a significant improvement of therapeutic standard, and PTL represents a model of therapeutic implementation and achievement in oncology.

\section{Conflict of Interests}

The authors declare that they have no conflict of interests.

\section{References}

[1] J. R. Austin, A. K. El-Naggar, and H. Goepfert, "Thyroid cancers-II: medullary, anaplastic, lymphoma, sarcoma, squamous cell," Otolaryngologic Clinics of North America, vol. 29, no. 4, pp. 611-627, 1996.

[2] R. K. Pedersen and N. T. Pedersen, "Primary non-Hodgkin's lymphoma of the thyroid gland: a population based study," Histopathology, vol. 28, no. 1, pp. 25-32, 1996.

[3] J. A. Singer, "Primary lymphoma of the thyroid," American Surgeon, vol. 64, no. 4, pp. 334-337, 1998.

[4] D. A. Wirtzfeld, J. S. Winston, W. L. Hicks Jr., and T. R. Loree, "Clinical presentation and treatment of non-Hodgkin's lymphoma of the thyroid gland," Annals of Surgical Oncology, vol. 8, no. 4, pp. 338-341, 2001.

[5] K. Aozasa, "Hashimoto's thyroiditis as a risk factor of thyroid lymphoma," Acta Pathologica Japonica, vol. 40, no. 7, pp. 459$468,1990$.

[6] I. Kato, K. Tajima, and T. Suchi, "Chronic thyroiditis as a risk factor of B-cell lymphoma in the thyroid gland," Japanese Journal of Cancer Research, vol. 76, no. 11, pp. 1085-1090, 1985.

[7] J. P. Logue, R. J. Hale, A. L. Stewart, M. B. Duthie, and S. S. Banerjee, "Primary malignant lymphoma of the thyroid: a clinicopathological analysis," International Journal of Radiation Oncology Biology Physics, vol. 22, no. 5, pp. 929-933, 1992.
[8] S. Widder and J. L. Pasieka, "Primary thyroid lymphomas," Current Treatment Options in Oncology, vol. 5, no. 4, pp. 307313, 2004.

[9] E. D. Williams, "Malignant lymphoma of the thyroid," Clinics in Endocrinology and Metabolism, vol. 10, no. 2, pp. 379-389, 1981.

[10] N. Niitsu, M. Okamoto, N. Nakamura, H. Nakamine, M. Bessho, and M. Hirano, "Clinicopathologic correlations of stage IE/IIE primary thyroid diffuse large B-cell lymphoma," Annals of Oncology, vol. 18, no. 7, pp. 1203-1208, 2007.

[11] E. A. Eisenhauer, P. Therasse, J. Bogaerts et al., "New response evaluation criteria in solid tumours: revised RECIST guideline (version 1.1)," European Journal of Cancer, vol. 45, no. 2, pp. 228 247, 2009.

[12] E. L. Kaplan and P. Meier, "Nonparametric estimation from incomplete observations," Journal of the American Statistical Association, vol. 53, no. 282, pp. 457-481, 1958.

[13] C. Thieblemont, A. Mayer, C. Dumontet et al., "Primary thyroid lymphoma is a heterogeneous disease," Journal of Clinical Endocrinology and Metabolism, vol. 87, no. 1, pp. 105-111, 2002.

[14] P. P. Carbone, H. S. Kaplan, K. Musshoff, D. W. Smithers, and M. Tubiana, "Report of the Committee on Hodgkin's disease staging classification," Cancer Research, vol. 31, no. 11, pp. 18601861, 1971.

[15] S. M. Ansell, C. S. Grant, and T. M. Habermann, "Primary thyroid lymphoma," Seminars in Oncology, vol. 26, no. 3, pp. 316-323, 1999.

[16] M. L. Klyachkin, R. W. Schwartz, M. Cibull et al., "Thyroid lymphoma: is there a role for surgery?" American Surgeon, vol. 64, no. 3, pp. 234-238, 1998.

[17] I. B. Rosen, S. B. Sutcliffe, M. K. Gospodarowicz et al., "The role of surgery in the management of thyroid lymphoma," Surgery, vol. 104, no. 6, pp. 1095-1099, 1988.

[18] C. Cha, H. Chen, W. H. Westra, and R. Udelsman, "Primary thyroid lymphoma: can the diagnosis be made solely by fineneedle aspiration?” Annals of Surgical Oncology, vol. 9, no. 3, pp. 298-302, 2002.

[19] D. Y. Greenblatt, T. Woltman, J. Harter, J. Starling, E. Mack, and $\mathrm{H}$. Chen, "Fine-needle aspiration optimizes surgical management in patients with thyroid cancer," Annals of Surgical Oncology, vol. 13, no. 6, pp. 859-863, 2006.

[20] K. J. Harrington, V. J. Michalaki, L. Vini et al., "Management of non-Hodgkin's lymphoma of the thyroid: the Royal Marsden Hospital experience," British Journal of Radiology, vol. 78, no. 929, pp. 405-410, 2005.

[21] T. J. Blair, R. G. Evans, S. J. Buskirk, P. M. Banks, and J. D. Earle, "Radiotherapeutic management of primary thyroid lymphoma," International Journal of Radiation Oncology Biology Physics, vol. 11, no. 2, pp. 365-370, 1985.

[22] R. Doria, J. F. Jekel, and D. L. Cooper, "Thyroid lymphoma. The case for combined modality therapy," Cancer, vol. 73, no. 1, pp. 200-206, 1994.

[23] L. Foppiani, V. Secondo, A. Arlandini, P. Quilici, M. Cabria, and P. Del Monte, "Thyroid lymphoma: a rare tumor requiring combined management," Hormones, vol. 8, no. 3, pp. 214-218, 2009.

[24] C. S. Portlock and S. A. Rosenberg, "Combination chemotherapy with cyclophosphamide, vincristine, and prednisone in advanced non Hodgkin's lymphomas," Cancer, vol. 37, no. 3, pp. 1275-1282, 1976. 
[25] T. P. Miller, S. Dahlberg, J. R. Cassady et al., "Chemotherapy alone compared with chemotherapy plus radiotherapy for localized intermediate- and high-grade non-Hodgkin's lymphoma," New England Journal of Medicine, vol. 339, no. 1, pp. 21-26, 1998.

[26] C. S. Ha, K. M. Shadle, L. J. Medeiros et al., "Localized nonHodgkin lymphoma involving the thyroid gland," Cancer, vol. 91, no. 4, pp. 629-635, 2001.

[27] F. Matsuzuka, A. Miyauchi, S. Katayama et al., "Clinical aspects of primary thyroid lymphoma: diagnosis and treatment based on our experience of 119 cases," Thyroid, vol. 3, no. 2, pp. 93-99, 1993.

[28] K. Aozasa, A. Inoue, K. Tajima, A. Miyauchi, F. Matsuzuka, and K. Kuma, "Malignant lymphomas of the thyroid gland: analysis of 79 patients with emphasis on histologic prognostic factors," Cancer, vol. 58, no. 1, pp. 100-104, 1986.

[29] A. Aziz Belal, A. Allam, A. Kandil et al., "Primary thyroid lymphoma: a retrospective analysis of prognostic factors and treatment outcome for localized intermediate and high grade lymphoma," American Journal of Clinical Oncology, vol. 24, no. 3, pp. 299-305, 2001.

[30] G. V. Burton, S. Atwater, M. J. Borowitz, and A. T. Huang, "Extranodal head and neck lymphoma. Prognosis and patterns of recurrence," Archives of Otolaryngology, vol. 116, no. 1, pp. 6973, 1990.

[31] C. Onal, Y. X. Li, R. C. Miller et al., “Treatment results and prognostic factors in primary thyroid lymphoma patients. A Rare Cancer Network study," Annals of Oncology, vol. 22, no. 1, pp. 156-164, 2011.

[32] R. W. Tsang, M. K. Gospodarowicz, S. B. Sutcliffe, J. F. G. Sturgeon, T. Panzarella, and B. J. Patterson, "Non-Hodgkin's lymphoma of the thyroid gland: prognostic factors and treatment outcome," International Journal of Radiation Oncology Biology Physics, vol. 27, no. 3, pp. 599-604, 1993. 


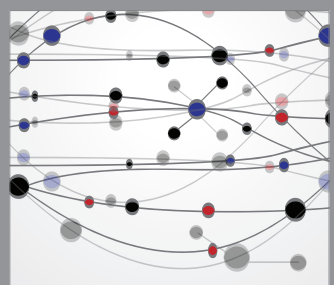

The Scientific World Journal
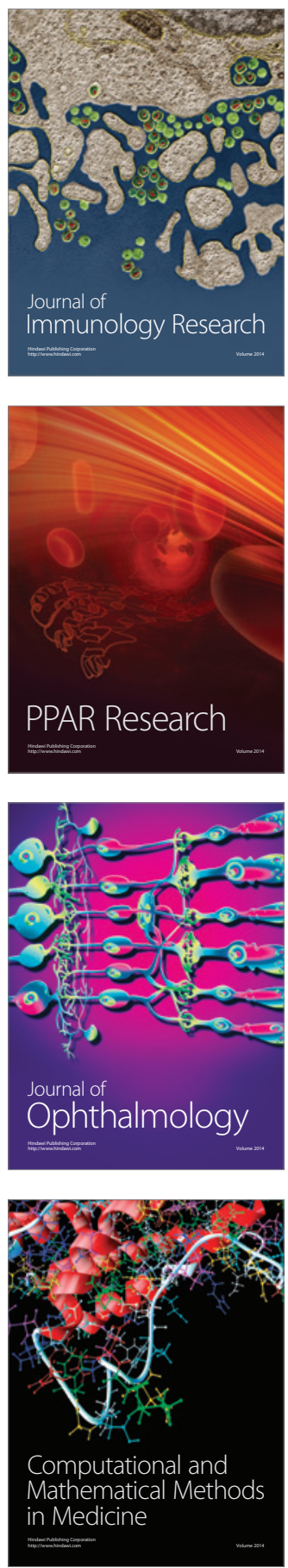

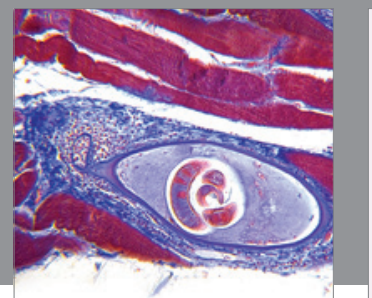

Gastroenterology

Research and Practice
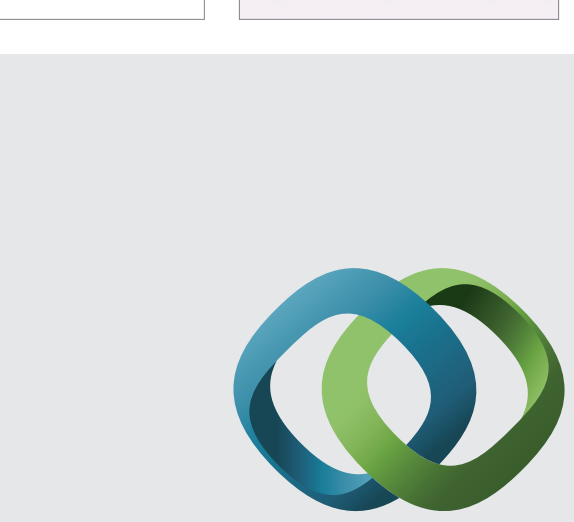

\section{Hindawi}

Submit your manuscripts at

http://www.hindawi.com
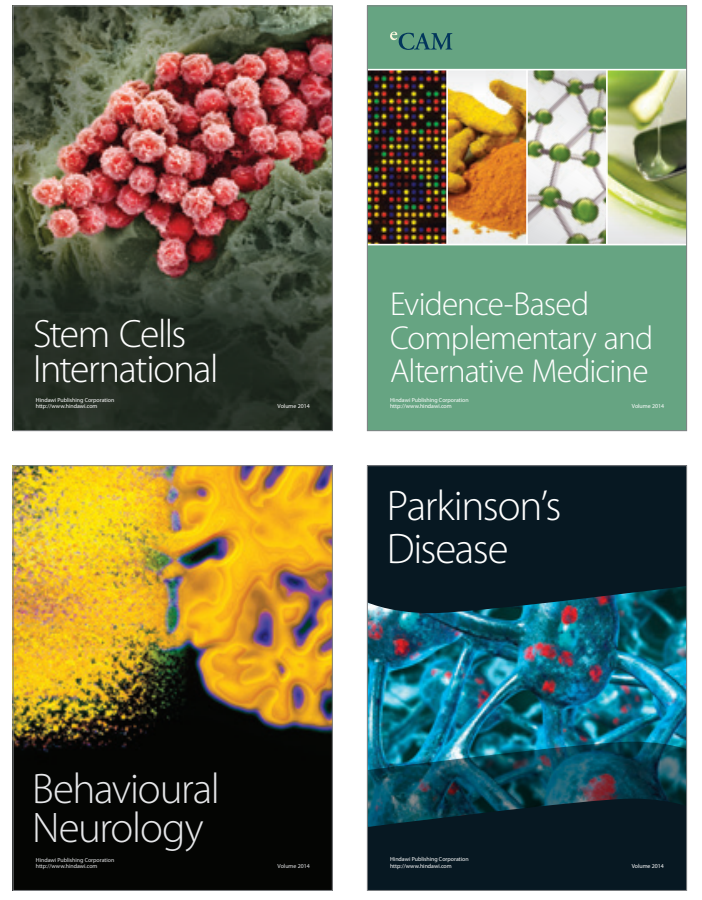
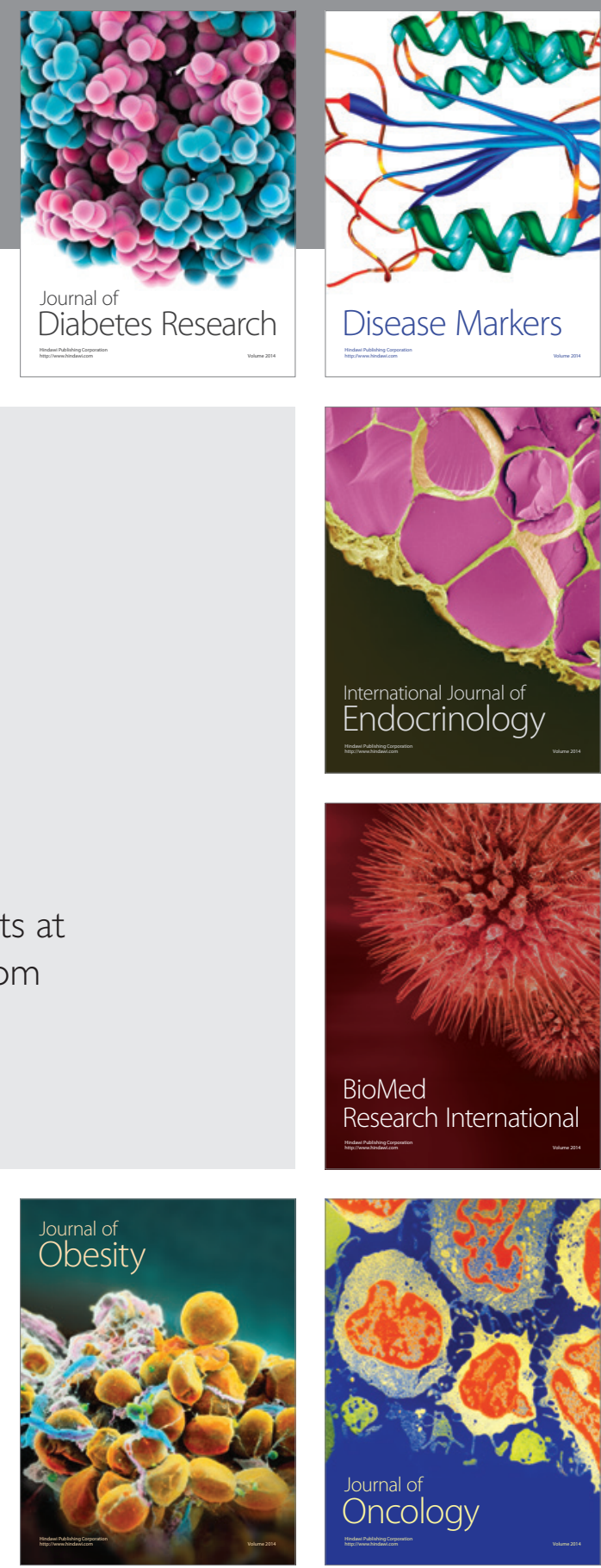

Disease Markers
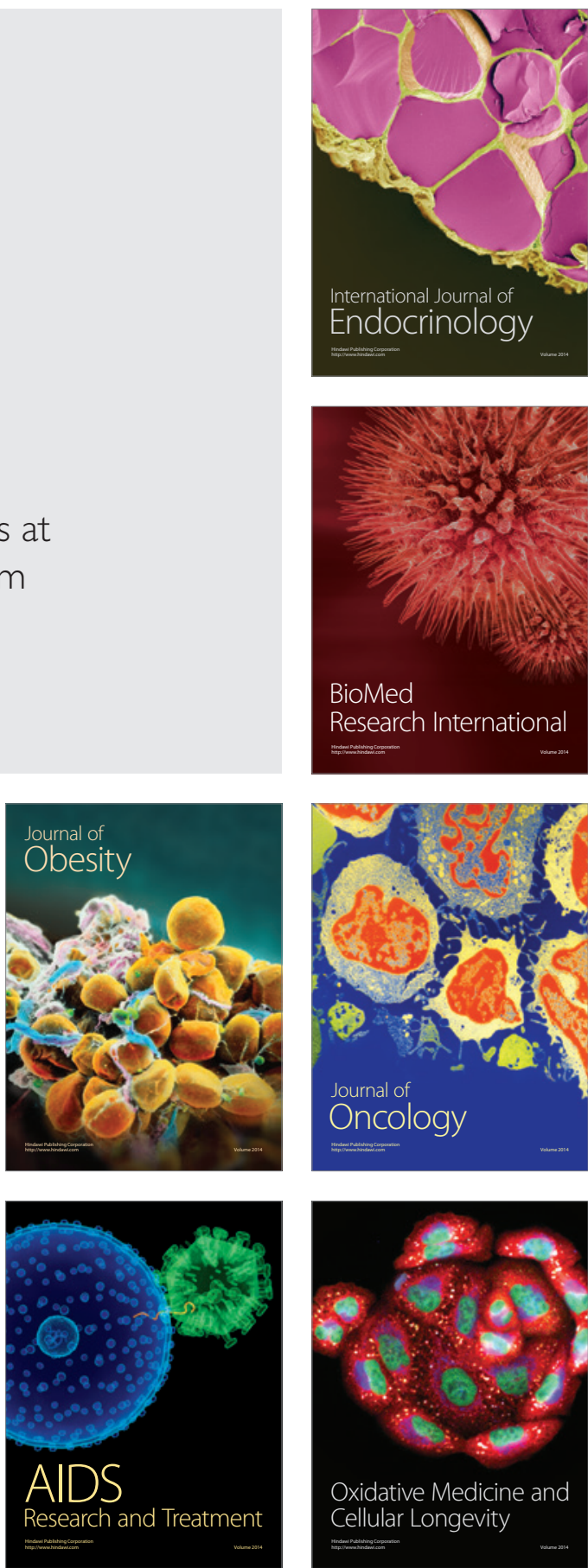